\title{
Cardiac Rupture
}

National Cancer Institute

\section{Source}

National Cancer Institute. Cardiac Rupture. NCI Thesaurus. Code C34668.

Tearing of the heart muscle. 